\title{
An improved test of the general relativistic effect of frame-dragging using the LARES and LAGEOS satellites
}

\author{
Ignazio Ciufolini ${ }^{1,2}$, Antonio Paolozzi ${ }^{3}$, Erricos C. Pavlis ${ }^{4}$, Giampiero Sindoni $^{3, \mathrm{a}}{ }_{\mathbb{C}}$, John Ries $^{5}$, Richard Matzner ${ }^{6}$, \\ Rolf Koenig ${ }^{7}$, Claudio Paris ${ }^{2,3}$, Vahe Gurzadyan ${ }^{8}$, Roger Penrose ${ }^{9}$ \\ ${ }^{1}$ Dip. Ingegneria dell'Innovazione, Università del Salento, Lecce, Italy \\ ${ }^{2}$ Centro Fermi-Museo Storico della Fisica e Centro Studi e Ricerche Enrico Fermi, Rome, Italy \\ ${ }^{3}$ Scuola di Ingegneria Aerospaziale, Sapienza Università di Roma, Rome, Italy \\ ${ }^{4}$ Joint Center for Earth Systems Technology, (JCET), University of Maryland, Baltimore, USA \\ ${ }^{5}$ Center for Space Research, University of Texas at Austin, Austin, USA \\ ${ }^{6}$ Theory Center, University of Texas at Austin, Austin, USA \\ ${ }^{7}$ Helmholtz Centre Potsdam, GFZ German Research Centre for Geosciences, Potsdam, Germany \\ ${ }^{8}$ Center for Cosmology and Astrophysics, Alikhanian National Laboratory and Yerevan State University, Yerevan, Armenia \\ ${ }^{9}$ Mathematical Institute, University of Oxford, Oxford, UK
}

Received: 27 September 2019 / Accepted: 8 October 2019 / Published online: 23 October 2019

(c) The Author(s) 2019

\begin{abstract}
We report the improved test of frame-dragging, an intriguing phenomenon predicted by Einstein's General Relativity, obtained using 7 years of Satellite Laser Ranging (SLR) data of the satellite LARES (ASI, 2012) and 26 years of SLR data of LAGEOS (NASA, 1976) and LAGEOS 2 (ASI and NASA, 1992). We used the static part and temporal variations of the Earth gravity field obtained by the space geodesy mission GRACE (NASA and DLR) and in particular the static Earth's gravity field model GGM05S augmented by a model for the 7-day temporal variations of the lowest degree Earth spherical harmonics. We used the orbital estimator GEODYN (NASA). We measured frame-dragging to be equal to $0.9910 \pm 0.02$, where 1 is the theoretical prediction of General Relativity normalized to its frame-dragging value and \pm 0.02 is the estimated systematic error due to modelling errors in the orbital perturbations, mainly due to the errors in the Earth's gravity field determination. Therefore, our measurement confirms the prediction of General Relativity for frame-dragging with a few percent uncertainty.
\end{abstract}

\section{General relativity, dragging of inertial frames and the objectives of the LARES space mission}

Einstein's gravitational theory of General Relativity is fundamental to understand our universe [1-4]. It has a number of outstanding experimental verifications [4-6], among which are the recent impressive LIGO laser interferometers direct

\footnotetext{
a e-mail: giampiero.sindoni@ uniroma1.it
}

detections of gravitational waves and observation of black holes, and of their collision, through the emission of gravitational waves $[7,8]$.

LARES (LAser RElativity Satellite) [9] is a laser-ranged satellite of ASI, the Italian Space Agency, dedicated to test General Relativity and fundamental physics, and to measurements of space geodesy and geodynamics. Among the tests of General Relativity, the main objective of LARES is a measurement of dragging of inertial frames, or frame-dragging, with an accuracy of a few percent. In addition to the test of frame-dragging, LARES, together with the LAGEOS (LAser GEOdynamcs Satellite of NASA) [11] and LAGEOS 2 (of ASI and NASA), has recently provided a test of the weak equivalence principle [10], at the foundations of General Relativity and other viable gravitational theories, with an accuracy of about $10^{-9}$, at a previously untested range between about 7820 and $12270 \mathrm{~km}$, and using previously untested materials of a tungsten alloy (the material of LARES) and aluminum-brass (the material of LAGEOS and LAGEOS 2). The orbital parameters and characteristics of the LARES and LAGEOS satellites are provided in the next section.

Frame-dragging [12] is an intriguing phenomenon of General Relativity: in Einstein's gravitational theory the inertial frames, which can only be defined locally (according to the equivalence principle $[1,2,4]$ ), have no fixed direction with respect to the distant stars but are instead dragged by the currents of mass-energy such as the rotation of a body, e.g., the rotation of the Earth (the axes of the local inertial frames are determined in General Relativity by local test-gyroscopes.) For a detailed description of such intriguing phenomenon and 
its fascinating astrophysical implications around spinning black holes, we refer to $[1,4,13-16]$. Here we just observe that the 2015-2018 detection of gravitational waves by the LIGO detectors $[7,8]$ are also based on computer simulations of the collision of spinning black holes and spinning neutron stars to form a spinning black hole. In such astrophysical processes, frame-dragging plays a key role [17].

The effect of frame-dragging on the orbit of a satellite is called the Lense-Thirring effect [18]. The orbital plane of a satellite may be thought of as a gyroscope (it would be a perfect gyroscope if the satellite were orbiting under the influence of a central gravitational force only, due to a spherically symmetric body). Indeed, the line of the nodes (intersection of the satellite's orbital plane with the equatorial plane of the central body $[19,20]$ ) has a frame-dragging shift described by the Lense-Thirring effect.

Between 2004 and 2016, frame-dragging was tested in a series of papers with an accuracy between about 10\% and 5\% using the two LAGEOS satellites [21-25] and the LARES and LAGEOS satellites [9,26]. In 2011, frame-dragging was tested using the Gravity Probe B experiment with an accuracy of about $20 \%$ [27]. It may be noted that GP-B measured the spin-spin effect due to frame-dragging, whereas LARES measured the spin-orbit frame-dragging effect. Thus, for example, gravitational theories with torsion (antisymmetric connection) may imply a different outcome for these two frame-dragging effects (for gravitational theories predicting frame-dragging effects different from General Relativity see, e.g., [28]). Furthermore the errors in each test have no commonality with each other, thus these tests complement each other and provide a more robust overall test of General Relativity.

\section{The LARES, LAGEOS, LAGEOS 2 and GRACE satellites and the method of analysis}

The LARES satellite [29] of ASI was successfully launched on 13th February 2012 using the VEGA launch vehicle (of ESA-ASI-ELV-AVIO). It has a semimajor axis of $7821 \mathrm{~km}$, orbital eccentricity of 0.0008 and inclination of $69.5^{\circ}$. Its mass is $386.8 \mathrm{~kg}$, its diameter $36.4 \mathrm{~cm}$ and is covered with 92 retro-reflectors (CCRs) reflecting back the laser pulses to precisely determine the range between the satellite and the laser-ranging stations on Earth with an uncertainty, for the best SLR (Satellite Laser Ranging) stations, of less than 1 $\mathrm{cm}$ [30]. The LAGEOS (NASA) [11] and LAGEOS 2 (ASINASA) satellites were respectively launched in 1976 and 1992. They are almost identical with a diameter of $60 \mathrm{~cm}$ and a mass of respectively 405.38 and $432 \mathrm{~kg}$. They are each covered with 426 CCRs. LAGEOS has a semimajor axis of $12270 \mathrm{~km}$, orbital eccentricity of 0.0045 and inclination of $109.84^{\circ}$, whereas LAGEOS 2 a semimajor axis of $12163 \mathrm{~km}$, orbital eccentricity of 0.0135 and inclination of $52.64^{\circ}$. The orbit of these three satellites can be determined with centimeter accuracy [30] using their observations by the International Laser Ranging Service (ILRS) [30] (their normal points have submillimeter accuracy) and orbital estimators such as GEODYN (NASA) [31], UTOPIA (CSR-UT) and EPOSOC (GFZ).

The twin GRACE (Gravity Recovery and Climate Experiment) satellites of NASA and DLR (Deutsche Forschunganstalt für Luft und Raumfahrt: German Aerospace Center) were launched in 2002. They have been orbiting at about 220 $\mathrm{km}$ apart, with a semimajor axis of $6856 \mathrm{~km}$, orbital eccentricity of 0.005 and inclination of $89^{\circ}$. GRACE, by accurately measuring the variations of the distance between its twin satellites using GPS and a microwave ranging system, allowed an unprecedented accuracy in the determination of the Earth gravity field and of its variations [32]. The GRACE science mission ended in October 2017. The GRACE FollowOn (GRACE-FO) space mission of NASA and GFZ (German Research Centre for Geosciences) [33] is the continuation of the GRACE mission. It was successfully launched in May 22,2018 . It will also test a new technology to dramatically improve the remarkable precision of its measurement system.

The main uncertainty in the measurement of the framedragging shift of the nodal line of an Earth satellite (the Lense-Thirring effect) is due to the uncertainty in the modelling of its nodal shift due to the deviations of the Earth gravity field from that of a perfect spherical body [19] and in particular due to the even zonal harmonics of the spherical harmonic expansion of the Earth gravity field. The even zonal harmonics describe the deviation from sphericity that are symmetric with respect to the Earth rotation axis and its equatorial plane; in fact the even zonal harmonics produce secular effects that cannot be separated from the secular LenseThirring effect using a single satellite. Thus, the largest uncertainties in modelling the nodal shift of an Earth satellite are those due to the Earth quadrupole moment, $J_{2}$, and to the next higher degree even zonal harmonic, $J_{4}$. Indeed, thanks to the GRACE determinations of the Earth gravity field and of its variations, the error in modeling the even zonal harmonics of degree strictly higher than four is at the level of a few percent only (see Sect. 3) [9,22, 25, 26,34].

The method to use $n$ observables, i.e., the nodal lines of $n$ laser-ranged satellites to eliminate the uncertainties in the first $n-1$ even zonal harmonics and to measure the Lense-Thirring effect, was proposed in [36], described in detail in [37] and reported in [21,22,25] to measure the Lense-Thirring effect, first using two satellites, LAGEOS and LAGEOS 2, and then using three satellites; LARES, LAGEOS and LAGEOS 2 [9].

In the analysis reported in the present paper, for the three unknowns given by the uncertainties in $J_{2}$ and $J_{4}$ and by the Lense-Thirring effect, we need three observables provided 
by the measured rates of the three nodal lines of LARES, LAGEOS and LAGEOS 2.

The combination that allows such measurement is $[9,26]$ :

$$
\begin{aligned}
\delta \dot{\Omega}^{\text {LAGEOS I }}+k_{1} \delta \dot{\Omega}^{\text {LAGEOS } 2}+k_{2} \delta \dot{\Omega}^{\text {LARES }} \\
=\mu\left(30.68+k_{1} 31.50+k_{2} 118.50\right) \text { mas } / y r \\
\quad+\text { other error } \cong \mu(50.18 \text { mas } / y r),
\end{aligned}
$$

where $\delta \dot{\Omega}$ are the residuals (observed minus calculated) of the nodal rates of LAGEOS, LAGEOS 2 and LARES, determined with GEODYN using their SLR data, the GRACE gravity field model GGM05S [35] together with a model of the time variation, and other up to date orbital perturbations, including tides (GOT4.10 tidal model [38]) and nongravitational orbital perturbations effects such as solar radiation pressure $[9,25]$.

In Eq. (1) $30.68 \mathrm{mas} / \mathrm{yr}, 31.50 \mathrm{mas} / \mathrm{yr}$ and $118.50 \mathrm{mas} /$ $y r$ are the Lense-Thirring nodal rates, respectively of LAGEOS, LAGEOS 2 and LARES, predicted by General Relativity. $k_{1}$ and $k_{2}$ are the coefficients to eliminate the uncertainties in $J_{2}$ and $J_{4}$ in measuring the Lense-Thirring effect, calculated by solving the three equations of the residual nodal rates of the three satellites. They are equal to unity for LAGEOS, and on average equal to $k_{1}=0.3448$ for LAGEOS 2, and $k_{2}=0.07291$ for LARES.

A source of error is due to the time-variations of the lowest harmonics of the Earth gravity field, both their rates and longperiodic variations, and their lunar and solar tidal changes. However, in the present analysis, in order to be able to reach an accuracy in the test of frame-dragging of about $2 \%$, we have applied the following techniques:

(1) we determined and used the precise values of $k_{1}$ and $k_{2}$ over each 15-day arc by using the precise but variable orbital elements of the three satellite's determined by GEODYN using the SLR data of the three satellites;

(2) over about one half of the period of our analysis, corresponding to the GRACE science phase, we applied the 7-day variations of the lowest Earth harmonics, compatible with the GGM05S model, directly in the orbital estimator GEODYN. Over the remaining period we applied the secular rates of the lowest harmonics determined by GRACE;

(3) finally, we applied the following new method to eliminate the uncertainties due to the main tidal perturbations. First, we observe that we have about 26 years of SLR data of both LAGEOS and LAGEOS 2, whereas we only have about 7 years of SLR data of LARES, since its launch date in 2012. Furthermore, the main tidal uncertainties in the combination of the residuals of the nodal rates of the three satellites, Eq. (1), are those affecting the nodes of LAGEOS and LAGEOS 2. The coefficient of the LARES nodal shift, $k_{2}=0.07291$, is much smaller than the coefficients of LAGEOS (unity) and LAGEOS $2 k_{1}=0.3448$. However, the largest tidal signals of LAGEOS and LAGEOS 2 are due to the K1 tide and have a period of about 1051 days and 571 days, respectively (i.e., they correspond to the well measured periods of the nodes of LAGEOS and LAGEOS 2 respectively). These long-period residual tidal perturbations can well be fitted for using the 26 years of SLR data of LAGEOS and LAGEOS 2. Therefore, using GEODYN, we processed 26 years of SLR data of LAGEOS and LAGEOS 2 to fit for the residual amplitudes of these two main tidal signals of the nodes of LAGEOS and LAGEOS 2, and then removed these two residual tidal amplitudes from their orbital residuals. We then combined the node residuals of LAGEOS, LAGEOS 2 and LARES, according to Eq. (1), over the last 7 years of their SLR data and, by a Fourier analysis of the residuals of the combination, Eq. (1), we found three main periodical signals affecting their combination (corresponding to tidal effects with well known frequencies due to the lunar and solar perturbations [39]). One of these residual tidal signals corresponds to the K1 tide of LARES with its short node period of about 211 days, well fitted for over 7 years of LARES SLR data. We then removed these three residual tidal effects from the combination of the orbital residuals of the three satellites and fitted for a secular trend.

After using this technique the combination of nodal rates of Eq. (1) became substantially unaffected by the nodal uncertainties due to the tidal errors, apart from smaller tidal signals with periods much shorter than the observation period of 7 years. Furthermore, the other variations in the Earth gravity field were well modelled using the GRACE data. In the next section, we show the Fourier analysis of the residuals of the combination, Eq. (1), before and after removal of the five main residual tidal signals (Figs. 3, 4). In Fig. 5 we also present the distribution of the combination of the residuals after removing the five largest tidal signals, which shows the behaviour of a Gaussian normal distribution.

\section{Results of the measurement of frame-dragging with LARES, LAGEOS and LAGEOS 2}

By fitting the combination of the residuals of LARES, LAGEOS and LAGEOS 2, Eq. (1), after removing the five main tidal residual signals of LAGEOS, LAGEOS 2 and LARES (using the technique explained in the previous Sect. 2), we found a value of frame-dragging equal to 0.9910 , the corresponding fit of the residuals is shown in Fig. 1. In Fig. 2 we show the cumulative combined residuals and their fit before removing the five main tidal residual signals of 


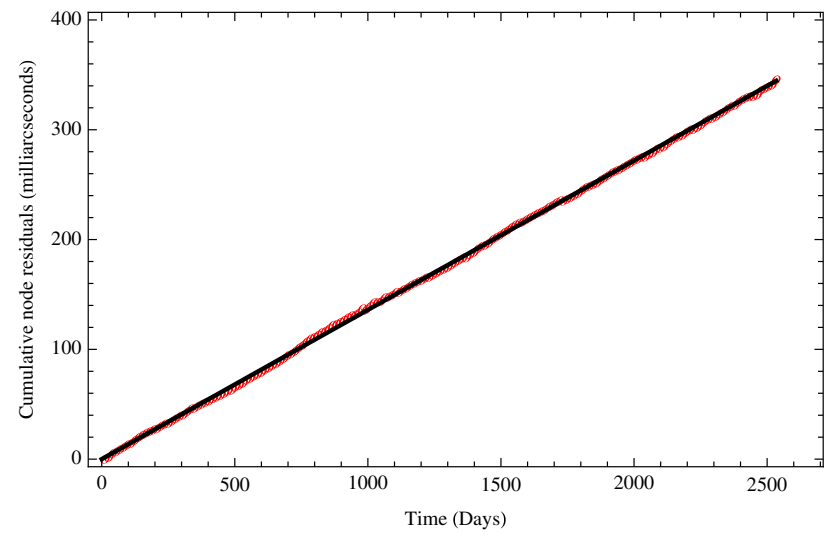

Fig. 1 Cumulative combined residuals of LARES, LAGEOS and LAGEOS 2 (shown in red), over about 7 years of orbital observations, after the removal the five main residual tidal signals. The solid black line is the fitted secular trend. The formal error of the fit is less than 0.001

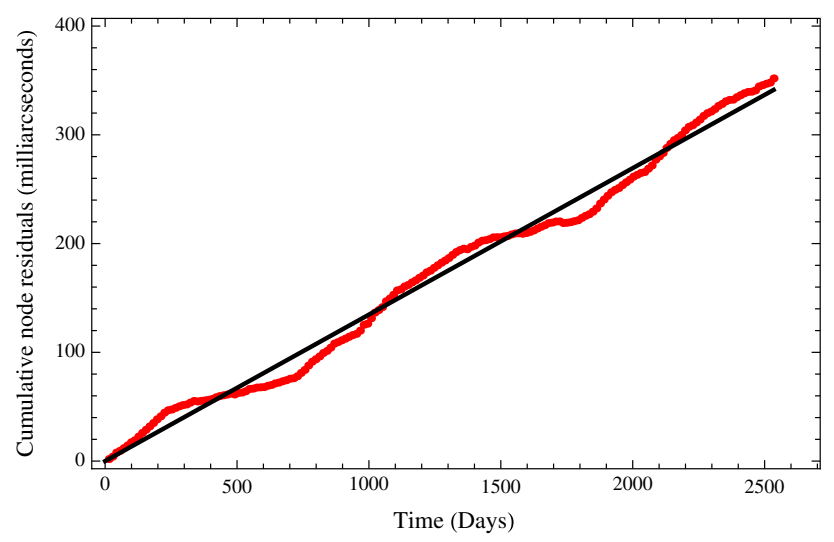

Fig. 2 Cumulative combined residuals of LARES, LAGEOS and LAGEOS 2 (shown in red), over about 7 years of orbital observations, fitted with a constant trend (shown with a solid black line)

LAGEOS, LAGEOS 2 and LARES. The final result for the frame-dragging effect shown in Fig. 1 is then:

$\mu=0.9910 \pm 0.02$.

Here $\mu=1$ is the value of frame-dragging normalized to its General Relativity value and 0.02 is the estimated total systematic error. This total systematic error can be estimated to be within about $2 \%$ and $3 \%$, whether or not one multiplies the published systematic errors of the static GGM05S gravity field for a safety factor of 2, i.e., depending on the estimation of the systematic errors of the gravity field model GGM05S. Indeed, by propagating the calibrated (including the published systematic uncertainties in their values) errors of GGM05S in the combination, Eq. (1), of the nodes, we found an error of $1.3 \%$ of the Lense-Thirring effect. Thus, by including other smaller errors due to the other gravitational and non-gravitational perturbations (see $[9,25]$ ), the RSS

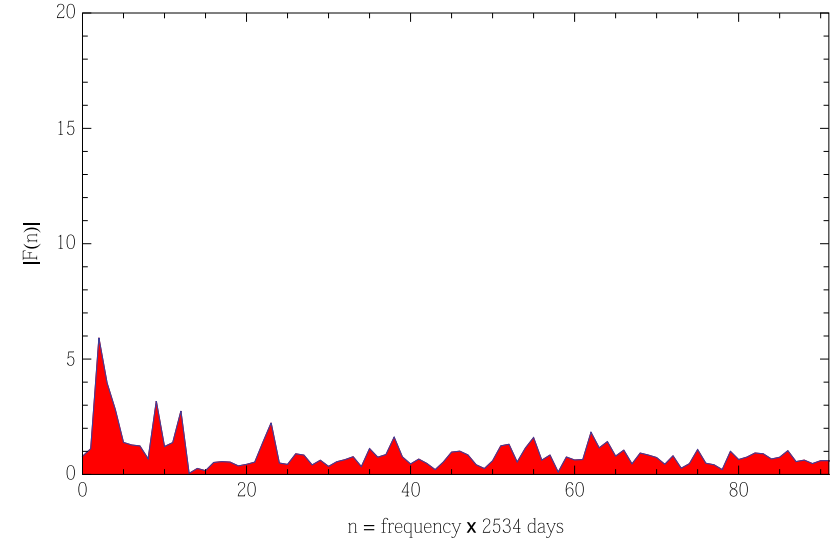

Fig. 3 Fourier analysis of the combined residuals of LARES, LAGEOS and LAGEOS 2 removing a constant trend, over about 7 years of orbital observations. In the horizontal axis is the frequency times 2534 days. In the vertical axis is the absolute value of the Discrete Fourier Transform $|F(n)|$

(Root Sum Squared) error is at the level of about 2\%. However, although the uncertainties for GGM05S have been carefully calibrated, this error calibration is statistical in nature. The errors are calibrated as a function of degree but cannot be expected to represent the error in any particular coefficient. To be more conservative, it is sensible to increase the uncertainty estimate of the static GGM05S gravity field by a factor of 2. In such a case, by multiplying for a factor 2 the calibrated errors of the static gravity field GGM05S, we get a total RSS error of about 3\%. The formal 1-sigma uncertainty of the fit of Fig. 1 is less than 0.001 .

In Fig. 3, we report the Fourier analysis of the combined residuals before removal of the five main tidal signals on the nodes of LAGEOS, LAGEOS 2 and LARES, and in Fig. 4, the Fourier analysis after successful removal of these five main tidal signals. Figure 4 shows that the main long-period tidal signals (with $n \lesssim 35$ ) were removed from the combination of the nodal residuals. The remaining higher frequencies do not significantly contribute to the secular trend fitted for over the observational period of 7 years. Figure 5 shows that the distribution of the residuals, after removal of the five main tidal signals, well approximates a normal Gaussian distribution.

We finally performed another relevant test to answer the following question: over which observational period (if any) does the measurement of frame-dragging with LARES, LAGEOS and LAGEOS 2 converge to within our errors to a final result? In other words over which observational period does the test of frame-dragging with LARES, LAGEOS and LAGEOS 2 becomes a stable test, substantially independent (i.e., within the total systematic error) in the increase of the period of data analysis? To answer this question we carried out the analysis and subsequent frame-dragging test over successive observational periods of time, each one increased 


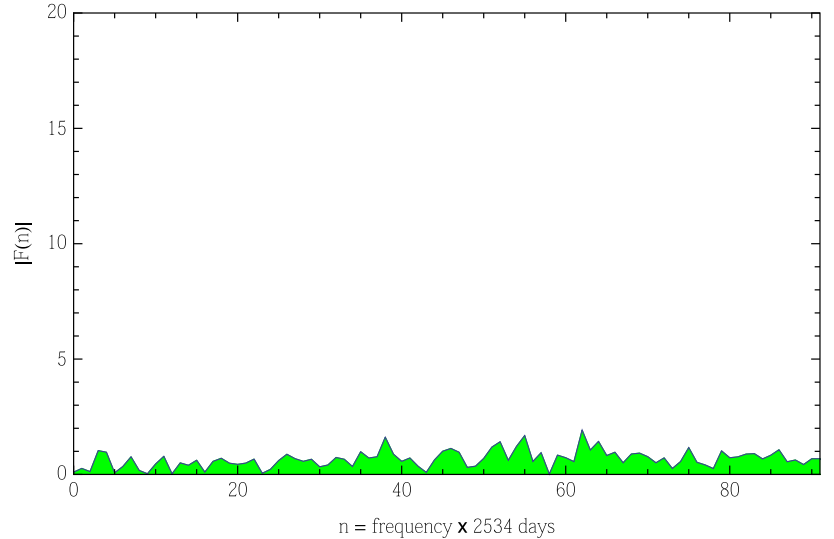

Fig. 4 Fourier analysis of the combined residuals of LARES, LAGEOS and LAGEOS 2, over about 7 years of orbital observations, after removal of the five main residual tidal signals and of a constant trend. In the horizontal axis is the frequency times 2534 days. In the vertical axis is the absolute value of the Discrete Fourier Transfom $|F(n)|$. The figure shows that there are no relevant long-period signals left after the removal of the six main tidal signals. The remaining higher frequencies $n$ do not significantly contribute to the secular trend fitted for over the observational period of 7 years

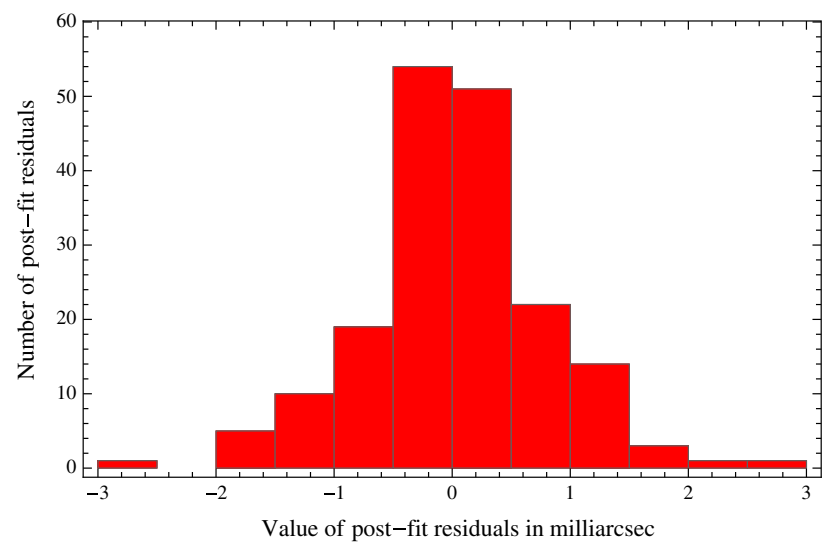

Fig. 5 Distribution of the post-fit combined residuals of LARES, LAGEOS and LAGEOS 2, over about 7 years of orbital observations. In the horizontal axis is the difference in milliarcsec of the residual of each arc, from the mean of the combined residuals. In the vertical axis is number of such combined residuals falling into each 0.5 milliarcsec bin. Here we have removed from the combined residuals the five main tidal signals (and the mean of the combined residuals). The distribution well approximates a Gaussian normal distribution, and supports the estimate of $2 \%$ accuracy determined by propagating the uncertainties of GGM05S combined with other smaller errors

by one residual (calculated over 14 days) over the previous period. The result is shown in Fig. 6. This figure clearly shows that after about three years of SLR observations, the measurement of frame-dragging is stable and clearly tends to its General Relativity value of 1 (normalized to its General Relativity value) well within a total systematic error of $2 \%$.

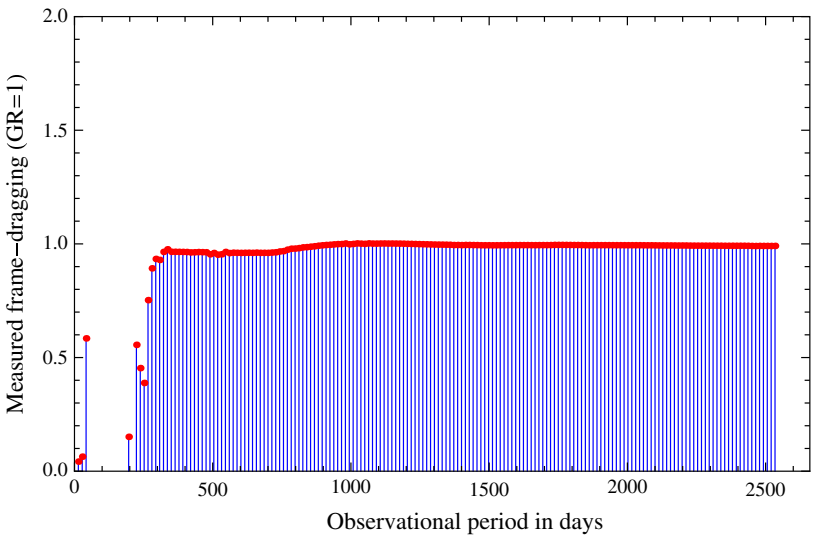

Fig. 6 Measurement of the Lense-Thirring effect using LARES, LAGEOS and LAGEOS 2 over increasing observational periods, i.e. each point of the curve was obtained by increasing the observational period of the previous point by 14 days, thus by increasing the total number of residuals by one with respect to the previous point. The curve clearly tends to its General Relativity value of 1 (normalized) well within the total systematic error of $2 \%$

\section{Conclusion}

We analyzed 7 years of SLR data of the satellite LARES (ASI), and 26 years of SLR data of LAGEOS (NASA) and LAGEOS 2 (ASI and NASA) using the Earth gravity field model GGM05S, together with the 7-day gravity field variations obtained by the space mission GRACE (NASA and DLR). Our analysis was performed with the NASA orbital estimator GEODYN.

By combining the residuals of these three laser-ranged satellites, we finally obtained a precise measurement of frame-dragging or Lense-Thirring effect. Our test fully confirms the prediction of Einstein's General Relativity: we obtained a measured value of frame-dragging of 0.9910 (to be compared to its General Relativity normalized value of 1 ), well within the total systematic error of $2 \%$. Indeed, the size of the total systematic error depends mainly on the considered size of the calibrated uncertainties in the even zonal harmonics of the Earth gravity field above degree 4. A total RSS error of about $2 \%$ corresponds to the propagation of the calibrated errors of GGM05S into the combination of the nodes of the three satellites (i.e., $1.3 \%$ due to the error in the even zonals plus other much smaller error sources).

Acknowledgements We gratefully acknowledge the support of the Italian Space Agency, Grants I/034/12/0, I/034/12/1 and 2015-021-R.0 and the International Laser Ranging Service for providing high-quality laser ranging tracking of the LARES satellites. E. C. Pavlis acknowledges the support of NASA Grants NNX09AU86G and NNX14AN50G. R. Matzner acknowledges NASA Grant NNX09AU86G and J. C. Ries the support of NASA Contract NNG17VI05C. 
Data Availability Statement This manuscript has associated data in a data repository. [Authors' comment: The SLR data of LAGEOS, LAGEOS 2 and LARES are available at: https://ilrs.cddis.eosdis.nasa. gov/data_and_products/data/npt/index.html.]

Open Access This article is distributed under the terms of the Creative Commons Attribution 4.0 International License (http://creativecomm ons.org/licenses/by/4.0/), which permits unrestricted use, distribution, and reproduction in any medium, provided you give appropriate credit to the original author(s) and the source, provide a link to the Creative Commons license, and indicate if changes were made.

Funded by SCOAP ${ }^{3}$.

\section{References}

1. C.W. Misner, K.S. Thorne, J.A. Wheeler, Gravitation (Freeman, San Francisco, 1973)

2. S. Weinberg, Gravitation and Cosmology: Principles and Applications of the General Theory of Relativity (Wiley, New York, 1972)

3. Y.B. Zel'dovich, I.D. Novikov, Relativistic Astrophysics (Chicago University Press, Chicago, 1971)

4. I. Ciufolini, J.A. Wheeler, Gravitation and Inertia (Princeton University Press, Princeton, 1995)

5. C.M. Will, The confrontation between general relativity and experiment. Living Rev. Relativ. 17, 4 (2014)

6. S. Turyshev, Experimental tests of general relativity: recent progress and future directions. Phys. Uspekhi 52, 1-27 (2009)

7. B.P. Abbott et al., Observation of gravitational waves from a binary black hole merger. Phys. Rev. Lett. 116, 061102 (2016)

8. B.P. Abbott et al., Multi-messenger observations of a binary neutron star merger. Astrophys. J. Lett. 848, 12 (2017)

9. I. Ciufolini, A. Paolozzi, E. Pavlis, J. Ries, R. Koenig, R. Matzner, G. Sindoni, The LARES Space Experiment: LARES Orbit, Error Analysis and Satellite Structure. General Relativity and John Archibald Wheeler, vol. 367 (Springer, Berlin, 2010), pp. 467-492

10. I. Ciufolini, R. Matzner, A. Paolozzi, E.C. Pavlis, G. Sindoni, J. Ries, V. Gurzadyan, R. Koenig, Satellite laser-ranging as a probe of fundamental physics. Sci. Rep. Nat. (2019) (in press)

11. S.C. Cohen, P.J. Dunn, LAGEOS scientific results. J. Geophys. Res. B 90(11), 9215 (1985)

12. A. Einstein, Letter to Ernst Mach, 25 June 1913. In: C. Misner, K.S. Thorne, J.A. Wheeler, Gravitation (Freeman, San Francisco, 1973) p. 544 (in ref. [1])

13. K.S. Thorne, R.H. Price, D.A. Macdonald, The Membrane Paradigm (Yale University Press, New Haven, 1986)

14. I. Ciufolini, Dragging of inertial frames. Nature 449, 41-47 (2007)

15. R.F. O'Connell, Gravito-magnetism in one-body and two-body systems: theory and experiments, in Proc. of the International School of Physics "Enrico Fermi", Course CLXVIII, Atom Optics and Space Physics, pp. 149-159

16. S. Kopeikin, E.B. Fomalont, Gravimagnetism, causality, and aberration of gravity in the gravitational light-ray deflection experiments. Gen. Relativ. Gravit. 39, 1583-1624 (2007)

17. Kip S. Thorne, Nobel Lecture, (NobelPrize.org, 2017), https:// www.nobelprize.org/prizes/physics/2017/thorne/lecture/.

18. J. Lense, H. Thirring, Uber den Einfluss der Eigenrotation der Zentralkörper auf die Bewegung der Planeten und Monde nach der Einsteinschen Gravitationstheorie, Phys. Z. , 19, 156-163, (1918). (See also English translation in: Gen. Relativ. Gravit., 16, 711-750, (1984))

19. W.M. Kaula, Theory of Satellite Geodesy (Blaisdell, Waltham, 1966)

20. S. Kopeikin, M. Efroimsky, G. Kaplan, Relativistic Celestial Mechanics of the Solar System (Wiley, New York, 2011)
21. I. Ciufolini, E.C. Pavlis, A confirmation of the general relativistic prediction of the Lense-Thirring effect. Nature 431, 958-960 (2004)

22. J.C. Ries, R.J. Eanes, M.M. Watkins, Confirming the framedragging effect with satellite laser ranging, in 16th International Workshop on Laser Ranging, Poznan, Poland, 13-17 October (2008)

23. J.C. Ries, Relativity in satellite laser ranging, in American Astronomical Society, IAU Symposium 261. Relativity in Fundamental Astronomy: Dynamics, Reference Frames, and Data Analysis (Virginia Beach, VA, USA, 27 April-1 May 2009)

24. R. König, B. Moreno-Monge, G. Michalak, Some aspects and perspectives of measuring Lense-Thirring with GNSS and geodetic satellites. in Second International LARES Science Workshop, Accademia dei Lincei, Rome, September (2012)

25. I. Ciufolini, E.C. Pavlis, J. Ries, R. Koenig, G. Sindoni, A. Paolozzi, H. Neumayer, Gravitomagnetism and its measurement with laser ranging to the LAGEOS satellites and GRACE Earth gravity models, In: General Relativity and John Archibald Wheeler, 367, 371434. SpringerVerlag GmbH, Berlino-DEU, (2010)

26. I. Ciufolini et al., A test of general relativity using the LARES and LAGEOS satellites and a GRACE Earth gravity model. Eu. Phys. J. C 76(3), 120 (2016)

27. C.W. Everitt et al., Gravity probe B: final results of a space experiment to test general relativity. Phys. Rev. Lett. 106, 22110 (2011)

28. T.L. Smith, A.L. Erickcek, R.R. Caldwell, M. Kamionkowski, Effects of Chern-Simons gravity on bodies orbiting the Earth. Phys. Rev. D 77(2), 024015 (2008)

29. A. Paolozzi, I. Ciufolini, C. Paris, G. Sindoni. LARES: A new satellite specifically designed for testing general relativity, Int. J. Aerosp. Eng., Article ID 341384, 9 pages, (2015)

30. M.R. Pearlman, C.E. Noll, E.C. Pavlis et al., The ILRS Approaching 20 years and planning for the future. J. Geod (2019). https:// doi.org/10.1007/s00190-019-01241-1

31. D.E. Pavlis et al., GEODYN Operations Manuals (Contractor Report, Raytheon, ITSS, Landover MD, 1998)

32. B.D. Tapley, S. Bettadpur, M. Watkins, C. Reigber, The gravity recovery and climate experiment: mission overview and early results. Geophys. Res. Lett. 31, L09607 (2004). https://doi.org/10. 1029/2004GL019920

33. https://gracefo.jpl.nasa.gov

34. I. Ciufolini, Monge B. Moreno, A. Paolozzi, R. Koenig, G. Sindoni, G. Michalak, E. Pavlis, Monte Carlo simulations of the LARES space experiment to test general relativity and fundamental physics. Class. Qauntum Grav. 30, 235009 (2013)

35. J. Ries, S. Bettadpur, R. Eanes, Z. Kang, U. Ko, C. McCullough, P. Nagel, N. Pie, S. Poole, T. Richter, H. Save, B. Tapley, Development and evaluation of the global gravity model GGM05, CSR-1602, Center for Space Research. The University of Texas at Austin (2016). https://doi.org/10.26153/tsw/1461

36. I. Ciufolini, A comprehensive introduction to the Lageos gravitomagnetic experiment: from the importance of the gravitomagnetic field in physics to preliminary error analysis and error budget. Int. J. Mod. Phys. A 4, 3083-3145 (1989)

37. I. Ciufolini, On a new method to measure the gravitomagnetic field using two orbiting satellites. Nuovo Cimento A 109, 1709-1720 (1996)

38. R.D. Ray, Precise comparisons of bottom-pressure and altimetric ocean tides. J. Geophys. Res. Oceans 118, 4570-4584 (2013). https://doi.org/10.1002/jgrc.20336

39. V.G. Gurzadyan et al., On the Earth's tidal perturbations for the LARES satellite. Eur. Phys. J. Plus 132, 548 (2017). https://doi. org/10.1140/epjp/i2017-11839-3 